\title{
Targeting cholesterol metabolism in glioblastoma: a new therapeutic approach in cancer therapy
}

\author{
Leila Pirmoradi, ${ }^{1}$ Nayer Seyfizadeh, ${ }^{2}$ Saeid Ghavami, ${ }^{\circledR 3,4,5,6}$ Amir A Zeki, ${ }^{7,8}$ \\ Shahla Shojaei ${ }^{9,10}$
}

For numbered affiliations see end of article.

\section{Correspondence to}

Dr Shahla Shojaei,

Department of Human Anatomy and Cell Sciences, Max Rady College of Medicine, Rady Faculty of Health Sciences, University of Manitoba, Winnipeg, Manitoba, Canada; shojaeis2019@gmail.com

Accepted 22 December 2018

Published Online First 14 February 2019
Check for updates

(C) American Federation for Medical Research 2019. No commercial re-use. See rights and permissions. Published by BMJ.

To cite: Pirmoradi L, Seyfizadeh N, Ghavami S, et al. J Investig Med 2019;67:715-719.

\section{ABSTRACT}

Glioblastoma multiforme (GBM) is the most aggressive malignant brain tumor known with a poor survival rate despite current advances in the field of cancer. Additional research into the pathophysiology of GBM is urgently needed given the devastating nature of this disease. Recent studies have revealed the unique cellular physiology of GBM cells as compared with healthy astrocytes. Intriguingly, GBM cells are incapable of de novo cholesterol synthesis via the mevalonate pathway. Thus, the survival of GBM cells depends on cholesterol uptake via low-density lipoprotein receptors (LDLRs) in the form of apolipoprotein-E-containing lipoproteins and ATP-binding cassette transporter A1 (ABCA1) that efflux surplus cholesterol out of cells. Liver $X$ receptors regulate intracellular cholesterol levels in neurons and healthy astrocytes through changes in the expression of LDLR and $A B C A 1$ in response to cholesterol and its derivatives. In GBM cells, due to the dysregulation of this surveillance pathway, there is an accumulation of intracellular cholesterol. Furthermore, intracellular cholesterol regulates temozolomide-induced cell death in glioblastoma cells via accumulation and activation of death receptor 5 in plasma membrane lipid rafts. The mevalonate pathway and autophagy flux are also fundamentally related with implications for cell health and death. Thus, via cholesterol metabolism, the mevalonate pathway may be a crucial player in the pathogenesis and treatment of GBM where our current understanding is still lacking. Targeting cholesterol metabolism in GBM may hold promise as a novel adjunctive clinical therapy for this devastating cancer.

\section{CHOLESTEROL METABOLISM IN CANCER}

Studies concerning the role of cholesterol in cancer have not been conclusive so far, and there is controversy regarding the correlation of dietary/intracellular cholesterol levels with different cancer types, ${ }^{1}$ While some studies showed positive correlations between increased serum cholesterol and the risk of special types of cancer like: prostate cancer, melanoma, non-Hodgkin's lymphoma, endometrial, and breast cancer, ${ }^{2}$ unexpectedly, other studies found no association. ${ }^{3}$ Also, it is not clear how much dietary cholesterol has an impact on the development of cancer. Some case reports showed positive correlations between the level of cholesterol consumption and some types of malignant tumors, ${ }^{4}$ while other evidence suggested no correlation. ${ }^{5}$

Normal cholesterol homeostasis leads to the production of the steroid hormones and contributes to the integrity of plasma membranes and intracellular signal transduction. This is mostly regulated by sterol regulatory element-binding protein transcription factor 2 and liver $\mathrm{X}$ receptors (LXRs). ${ }^{6}$

The Cancer Genome Atlas (TCGA) analysis revealed a prognostic signature of cholesterol synthesis genes. It showed an association between reduced survival of patients with sarcoma, acute myeloid leukemia, and melanoma with upregulation of the mevalonate pathway (negative correlation). However, in lower grade gliomas, the correlation was positive. Thus, there appears to be differential correlation between mevalonate pathway activation and prognostic outcomes that depends on the type of cancer. ${ }^{7}$ Moreover, constitutive activation of numerous oncogenic pathways, like receptor tyrosine kinase (RTK)/ RAS GTPase (RAS) and phosphatidylinositol 3-kinase (PI3K)/Akt/mammalian Target of Rapamycin (mTOR), induces cholesterol biosynthesis. ${ }^{8}$ The loss of $\mathrm{p} 53$ function deregulates the mevalonate pathway in cancer cells, which suggests a role for $\mathrm{p} 53$ in the modulation of cholesterol synthesis genes. ${ }^{9}$ In addition, dysregulation of mitochondrial cholesterol levels was associated with cancer cell survival where elevated mitochondrial cholesterol level induces resistance to apoptotic signals through STAR and STARD3, which regulate cholesterol uptake into mitochondria. ${ }^{10}$ Furthermore, cancer cell survival is regulated by ATP-binding cassette transporter A1 (ABCA1), a cell membrane-bound cholesterol efflux transporter, by which decreased ABCA1 activity increases mitochondrial cholesterol levels and subsequently increases cancer cell survival. ${ }^{11}$

Overall, while not conclusive, it appears that modulation of cholesterol homeostasis is an important contributor to cancer development. In this regard, many ideas have been developed to target cholesterol synthesis and/or transport or intestinal absorption to disrupt cholesterol homeostasis in cancer cells. ${ }^{12-14}$ 


\section{Uncoupling the nuclear receptor liver $\mathbf{X}$}

Liver X receptors (LXRs) are members of the nuclear receptor superfamily of transcription factors. Two isoforms of LXRs have been identified (LXR $\alpha$ and LXR $\beta$ ) that are known as whole-body cholesterol sensors and on activation through binding to their cognate ligand leads to a net elimination of cholesterol from the body. ${ }^{15}$ LXRs do that via induction of cholesterol hepatic excretion, ${ }^{16}$ decrease of its cellular uptake, and reduction of its absorption. ${ }^{17}$ Activation of LXRs is dependent on their coupling with retinoic acid receptors (RXRs) subsequent to binding to oxysterols that are mono-oxygenated derivatives of cholesterol. Oxysterols function as a negative feedback regulator of cholesterol homeostasis and biosynthesis by mediating increased expression of cholesterol efflux genes with decreased expression of genes needed for its synthesis and uptake. ${ }^{18}$

The high growth rates of cancer cells is a demanding process. It requires accumulation of intracellular cholesterol and reduction in oxysterol content that consequently results in LXR transcriptome downregulation. ${ }^{19}$ This homeostatic pathway is characterized by reduced oxysterol synthetic/ anabolic pathways and enhanced catabolic and secretory cascades. Based on this oxysterol hypothesis, under conditions of low levels of intracellular sterols, cholesterol synthesis and uptake are promoted, whereas cholesterol efflux is repressed. In addition, oxysterol synthesis is inhibited, and its catabolism and secretion are increased. The converse is true in situations of high intracellular sterol levels. ${ }^{20}$ Overall, high levels of intracellular cholesterol with low levels of oxysterols along with downregulated LXR/ RXR transcriptional activity support the high proliferation rates of cancer cells. Thus, rapidly growing cancer cells uncouple cholesterol and oxysterol homeostasis through downregulated LXR transcriptional activity, in order to increase intracellular cholesterol content. ${ }^{2122}$

Cellular cholesterol content is mediated by the integrated activities of synthesis, uptake, and efflux, which are impaired in conditions of high-rate cell proliferation. Thus, targeting pathways involved in cholesterol homeostasis could be a potential therapeutic approach in cancer treatment. Recent reports indicate that LXR significantly impacts cell growth arrest and the activation of apoptotic pathways. This observation supports the idea that LXRs have potential antitumor effects by modulating sterol metabolism. Therefore, targeting LXRs might be a novel approach in treating tumors given their ability to regulate cholesterol hemostasis and suppress the cell cycle. ${ }^{23-25}$

\section{Cholesterol metabolism in glioblastoma multiforme (GBM)}

Despite advanced chemotherapy, surgical interventions, and in-depth investigations to develop effective treatments, GBM is the most common malignant tumor of the central nervous system with a life expectancy of fewer than 2 years after diagnosis. ${ }^{26}$ The cholesterol pathway has emerged as a potentially new target for GBM amenable to targeted pharmacologic treatment. ${ }^{27}$ Cholesterol uptake is a crucial step for growth and survival for GBM cells. ${ }^{27}$ Therefore, defects in cholesterol biosynthesis and its downstream signaling can lead to cancer and neurodegenerative diseases, and therefore, represents a potential target for treatment. ${ }^{28}$
Levels of cholesterol in the brain are high and represent $20 \%-25 \%$ of total body cholesterol. However, the dynamics of the brain cholesterol pool and its metabolism is distinct from other organs due to the inability of peripheral cholesterol to cross the blood-brain barrier (BBB). ${ }^{27}$ Thus, the majority of cholesterol in the brain is synthesized via de novo biosynthesis by astrocytes and delivered to neurons within high-density lipoproteins containing apolipoprotein $\mathrm{E}$ (Apo-E). ${ }^{29}$ Unlike healthy astrocytes, the metabolic needs of GBM cells are supplied mainly by exogenously rather than endogenously synthesized cholesterol. ${ }^{27}$ As 3-hydroxy-3-methyl-glutaryl-coenzyme A (HMG-CoA) reductase inhibitors ('statins') inhibit de novo synthesis of cholesterol, it is not surprising that GBM cells are not as sensitive as healthy astrocytes to statins. ${ }^{27}$

LXR $\alpha$ and LXR $\beta$, the main regulators of cholesterol homeostasis, have an important role in lipoprotein uptake in brain cells. ${ }^{27}$ LXR ligands cause their heterodimerization with RXRs (LXR:RXR) and subsequent transcriptional activation of genes responsible for propagating lipid transport between neurons and glial cells. ${ }^{27}$ Oxysterols, which are synthesized from cholesterol by neurons and healthy astrocytes, function as endogenous LXR ligands. Receptor

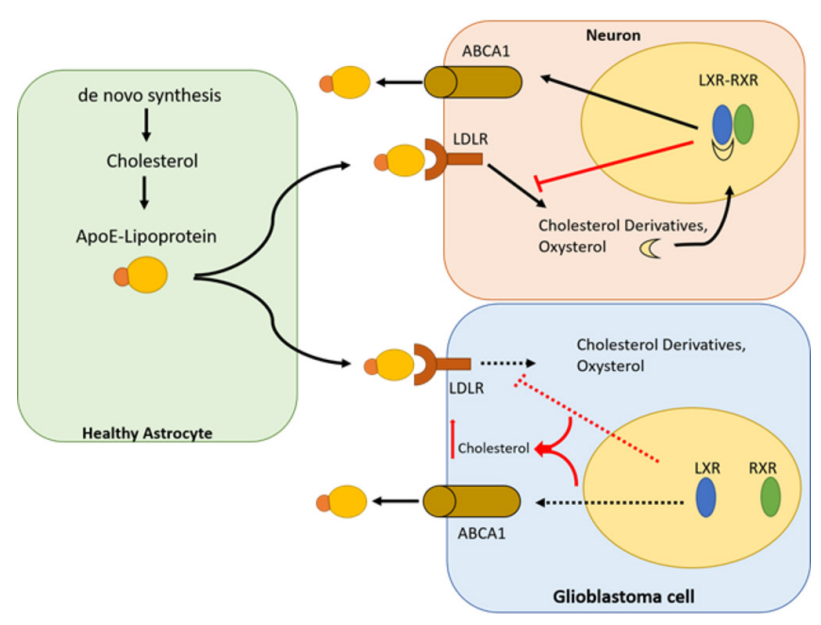

Figure 1 Neurons and glioblastoma cells rely on astrocytes to provide cholesterol via de novo synthesis. Neurons and glioblastoma cells do not use de novo synthesis of cholesterol and are dependent on uptake of exogenous cholesterol. They take up cholesterol produced and secreted by astrocytes in ApoE-containing lipoproteins. Oxysterol and cholesterol derivatives produced in neurons following cholesterol uptake and metabolism are physiological agonists for LXR. Activation of LXR results in its dimerization with RXR and subsequent increase in $A B C A 1$ cassette protein expression, the main exporter of cholesterol in the form of Apo-E-containing lipoproteins. It also inhibits the expression of the LDL receptor resulting in a decrease in cholesterol uptake, thereby regulating the level of intracellular cholesterol. In GBM cells, these cholesterol regulatory and surveillance mechanisms are disrupted. Thus, following the uptake of cholesterol in Apo-E-containing lipoproteins and the metabolism of cholesterol in GBM cells, oxysterol and other cholesterol derivatives are unable to activate LXR leading to the accumulation of intracellular cholesterol. ABCA1, ATP-binding cassette transporter A1; Apo-E, apolipoprotein E; GBM, glioblastoma multiforme; LDL, low-density lipoprotein receptor; $L X R$, liver $X$ receptors; $R X R$, retinoid $X$ receptors. 
activation diminishes the levels of cellular cholesterol in neurons and healthy astrocytes by two mechanisms: (1) increased cholesterol efflux through ABCA1 sterol transporters and (2) reduced cholesterol uptake via LDL receptor (LDLR) degradation. ${ }^{30}$

GBM cells are unable to produce sufficient endogenous LXR ligands thereby promoting exogenous cholesterol uptake. Villa et al showed that levels of LDLR and LDL uptake were higher, and LXR ligands were lower in GBM cells as compared with healthy astrocytes. ${ }^{27}$ This suggests that supplementation with exogenous LXR ligands that incorporate efficiently within the affected area of brain tissue may represent a potentially new anticancer strategy for GBM (figure 1). ${ }^{27}$

LXR-623 is a synthetic LXR agonist initially used for atherosclerosis treatment, but its ability to cross the BBB resulted in a study on glioblastoma cells that confirmed a killing effect by LXR-623 on U87EGFRvIII and GBM39 cells in vitro. ${ }^{27}$ In addition, LXR-623 showed anti-GBM activity in a xenograft model of intracranial GBM. ${ }^{27}$ Since GBM survival depends on cholesterol, the depletion of cellular cholesterol by LXR-623 leads to cell death. ${ }^{27}$ Mechanistically, treatment with LXR-623 also suppressed LDLR expression and enhanced expression of the ABCA1 cholesterol efflux transporter, which then induced substantial apoptosis via activation of the LXR $\beta$ isoform. ${ }^{27}$

There are other approaches involving cholesterol metabolism known in the GBM field. Phytol and retinol kill glioblastoma cells by interfering with fatty acid and cholesterol metabolism. The higher levels of lipids in GBM cells, necessary for their survival is partly due to involvement of an oncogenic pathway called epidermal growth factor receptor (EGFR)/PI3K/Akt. This pathway increases levels of intracellular lipids and upregulates sterol regulatory element-binding protein (SREBP-1) which, in turn, enhances lipid uptake. ${ }^{31}$ Therefore, SREBP-1 suppression can also lead to GBM cell death. ${ }^{32}$ Phytol alters the expression of SREBP-1 and sterol regulatory element-1, which are important constituents for regulation of cholesterol metabolism. ${ }^{31}$ Expression of fatty acid synthase (FAS) protein, a key tumor lipogenesis enzyme, is regulated by SREBP-1. Phytol and retinol inhibit SREBP-1 and FAS expression in U87MG, A172, and T98G cell lines of human glioblastoma. ${ }^{31}$ Thus, targeting GBM cells with phytol and retinol represents an alternative strategy in the fight against GBM.

Archazolid B is another drug used in the treatment of GBM. By affecting specific genes, its application results in the accumulation of free cholesterol within intracellular organelles and effectively depletes usable cellular cholesterol. Although archazolid B upregulates LDLR, which increases extracellular cholesterol uptake, it hampers the action of V-ATPase due to a proton transport defect. This leads to associated increases in lysosomal $\mathrm{pH}$, thereby preventing cholesterol recycling. ${ }^{33}$ This 'sequestration' of cholesterol makes it effectively absent for use by GBM cells. It is tempting to think that combinations of the above approaches to control cholesterol hemostasis in GBM cells could lead to a more robust therapeutic strategy in GBM than what is currently available.

In general, drug resistance is a serious problem in cancer therapy targeting cell death, especially in GBM. In light of currently applied strategies targeting GBM, it is important to realize that levels of intracellular cholesterol in GBM cells determine their resistance to chemotherapeutic drugs, such as temozolomide (TMZ), particularly in long-term treatment approaches. ${ }^{34}$ Although the distinct lipid metabolism of GBM cells has been reported, the role of intracellular cholesterol flux in TMZ-induced cell death requires additional investigation.

According to Yamamoto et al, in TMZ-resistant U251 cells, intracellular cholesterol levels are lower than non-resistant ones. They showed that decreasing intracellular cholesterol in non-resistant U251 cells using methyl- $\beta$-cyclodextrin increased cell viability, while adding cholesterol decreased it. ${ }^{13} 34$ This effect occurred via accumulation and activation of death receptor 5 (DR5) in cell plasma membrane lipid rafts. DR5 is a protein of the tumor necrosis factor receptor family, which is located in cell membranes and impacts cell death partially through caspase-dependent signaling. Lipid rafts that have high cholesterol microdomains regulate the molecular processes of DR5 action, and increased intracellular cholesterol augments activation of DR5 in the lipid rafts of GBM cells. ${ }^{34}$ DR5 also evokes caspase- 8 activity in response to TMZ. ${ }^{34}$ Soluble cholesterol enters cell membranes, which promotes signaling involved in DR5 activation. ${ }^{34}$ Therefore, using TMZ as a DR 5 trigger along with soluble cholesterol could potentially serve as combination therapy to treat GBM. ${ }^{34}$ Furthermore, the authors

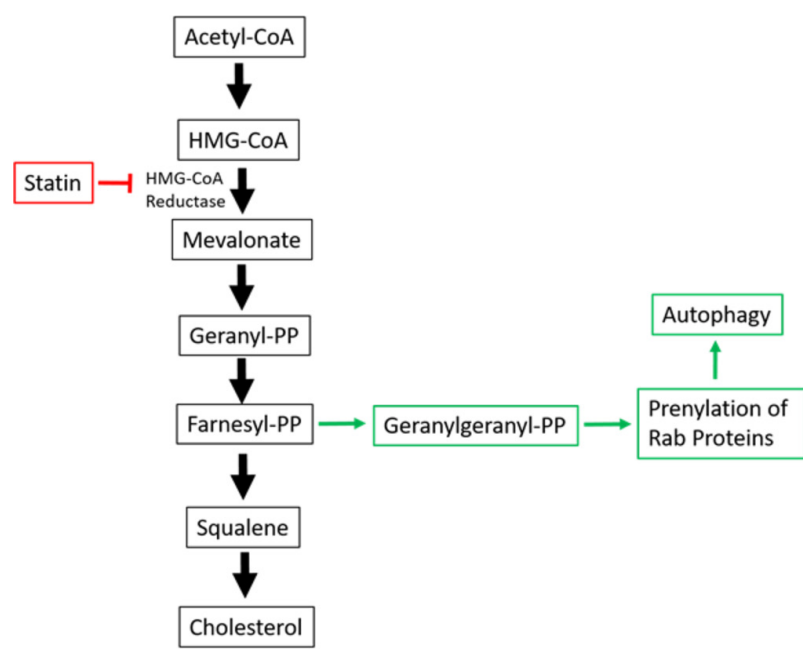

Figure 2 Role of the mevalonate pathway in autophagy. The mevalonate cascade is the de novo pathway for cholesterol biosynthesis in cells. It is also responsible for the production of the isoprenoids such as geranylgeranyl-pyrophosphate (geranylgeranyl-PP) that play an important role in the prenylation of the superfamily of Ras-like GTPase proteins known as the Rab family. Rab GTPases are involved in vesicular trafficking where Rab11 and Rab7 are critical components for autophagosome formation and autophagosome-lysosome fusion. Statins lower cholesterol due to inhibition of HMG-CoA reductase, the ratelimiting enzyme in cholesterol biosynthesis, but they also reduce mevalonate and the downstream isoprenoids. Thus, inhibition of the mevalonate pathway by statins results in decreased prenylation of Rab11 and Rab7 and subsequent inhibition of autophagy flux. HMG-CoA, 3-hydroxy-3-methyl-glutaryl-coenzyme A; PP, pyrophosphate. 
also showed that pharmacologically relevant concentrations of HMG-CoA reductase inhibitors (the 'statins') reduced GBM cell death induced by TMZ. ${ }^{34}$ Contrary to their findings, we reported potentiation of TMZ-induced apoptosis in GBM cells by simvastatin; however, in line with their findings, this effect was independent of cholesterol levels. ${ }^{35}$

This effect of statins may be due to regulation of autophagy by the mevalonate cascade. ${ }^{36}{ }^{37}$ We know that the mevalonate pathway modulates autophagy through geranylgeranylation of the small GTPase molecule Rab11 (figure 2) ${ }^{3638}$ Small GTPase Rab11 is a member of the Rab protein family and is involved in intracellular vesicular trafficking ${ }^{39}$ and belongs to the superfamily of Ras-like GTPases whose function is regulated by geranylgeranylation. ${ }^{39}$ Rab11 plays a pivotal role in autophagosome formation, ${ }^{40}$ and both Rab11 and Rab7 are necessary for autophagosome-lysosome fusion. ${ }^{39} 40$ The mevalonate pathway and Rab11 are mandatory components of basal autophagy flux due to their regulation of autophagosome formation and autophagosome-lysosome fusion ${ }^{36}$; however, the mevalonate pathway has no effect on lysosome function. ${ }^{36}$

Due to this crosstalk between autophagy and apoptosis, ${ }^{41}$ inhibition of the mevalonate pathway accompanies autophagy inhibition and consequently leads to apoptotic cell death. ${ }^{37}$ Our own research showed similar results where inhibition of the mevalonate pathway sensitized glioblastoma cells to TMZ-induced apoptosis through inhibition of autophagy flux and autophagosome-lysosome fusion. ${ }^{35}$ Of note, a big cohort study conducted on patients with different types of cancers reported increased survival rate of patients with long-term consumption of statins, ${ }^{3}$ and another subsequent study in glioblastoma patients showed the same results. ${ }^{43}$ Thus, the cholesterol-lowering drugs, statins, may potentially serve as a new therapeutic approach for combination therapy in glioblastoma. ${ }^{44}$

In summary, a deeper understanding of the role of cholesterol metabolism and transport in GBM cells and the underlying mechanisms of cholesterol-related drug resistance will be useful in improving current treatment strategies for patients with GBM. Mevalonate biology and autophagy are intimately linked at a more fundamental cellular level, where both pathways are amenable to pharmacologic manipulation. Furthermore, such an understanding could lead to the development of targeted and more effective therapies for GBM patients, a devastating disease with poor prognosis.

\footnotetext{
Author affiliations

${ }^{1}$ Department of Physiology and Pharmacology, Faculty of Medicine, Kurdistan University of Medical Sciences, Sanandaj, Kurdistan, Iran

${ }^{2}$ Stem Cell Research Center, Tabriz University of Medical Sciences, Tabriz, Iran ${ }^{3}$ Department of Human Anatomy and Cell Sciences, Max Rady College of Medicine, Rady Faculty of Health Sciences, University of Manitoba, Winnipeg, Manitoba, Canada

${ }^{4}$ Biology of Breathing, Children Hospital Research Institute of Manitoba, University of Manitoba, Winnipeg, Manitoba, Canada

${ }^{5}$ Research Institute in Oncology and Hematology, Cancer Care Manitoba, University of Manitoba, Winnipeg, Manitoba, Canada

${ }^{6}$ Health Policy Research Center, Institute of Health, Shiraz University of Medical Sciences, Shiraz, Fars, Iran

${ }^{7}$ Division of Pulmonary, Critical Care, and Sleep Medicine, University of California Davis, Sacramento, California, USA

${ }^{8}$ Center for Comparative Respiratory Biology and Medicine, University of California, Davis, School of Medicine, Davis, California, USA
}

${ }^{9}$ Department of Human Anatomy and Cell Sciences, Max Rady College of Medicine, Rady Faculty of Health Sciences, University of Manitoba, Winnipeg, Manitoba, Canada

${ }^{10}$ Department of Clinical Biochemistry, School of Pharmacy and Pharmaceutical Sciences, Isfahan University of Medical Sciences, Isfahan, Isfahan, Iran

Contributors SS conceived the idea and manuscript, LP and NS drafted the manuscript, SS and SG approved the final version of the article. AAZ added original content then also reviewed, edited and approved the final version of the manuscript.

Funding SG was supported by Research Manitoba New Investigator Operating grant and Health Science General Operating grant. SS was supported by MITACS accelerate (formerly called Mathematics of Information Technology and Complex Systems) postdoctoral fellowship. AAZ was supported by U.C. Davis PI Bridge Funds and Tobacco-Related Disease Research Program.

\section{Competing interests None declared.}

Provenance and peer review Not commissioned; externally peer reviewed.

\section{REFERENCES}

1 Kuzu OF, Noory MA, Robertson GP. The role of cholesterol in cancer. Cancer Res 2016;76:2063-70.

2 Allott EH, Howard LE, Cooperberg MR, et al. Serum lipid profile and risk of prostate cancer recurrence: results from the SEARCH database. Cancer Epidemiol Biomarkers Prev 2014;23:2349-56.

3 Nielsen SF, Nordestgaard BG, Bojesen SE. Statin use and reduced cancerrelated mortality. N Engl J Med 2012;367:1792-802.

4 Chen H, Qin S, Wang M, et al. Association between cholesterol intake and pancreatic cancer risk: evidence from a meta-analysis. Sci Rep 2015;5:8243.

$5 \mathrm{Hu}$ J, La Vecchia C, de Groh M, et al. Dietary cholesterol intake and cancer. Ann Oncol 2012;23:491-500.

6 Ikonen E. Cellular cholesterol trafficking and compartmentalization. Nat Rev Mol Cell Biol 2008;9:125-38

7 Weinstein JN, Collisson EA, Mills GB, et al. The cancer genome atlas pan-cancer analysis project. Nat Genet 2013;45:1113-20.

8 Dong F, Mo Z, Eid W, et al. Akt inhibition promotes ABCA1-mediated cholesterol efflux to ApoA-I through suppressing mTORC1. PLOS One 2014;9:e113789.

9 Sorrentino G, Ruggeri N, Specchia V, et al. Metabolic control of YAP and TAZ by the mevalonate pathway. Nat Cell Biol 2014;16:357-66.

10 Vassilev B, Sihto H, Li S, et al. Elevated levels of StAR-related lipid transfer protein 3 alter cholesterol balance and adhesiveness of breast cancer cells: potential mechanisms contributing to progression of HER2-positive breast cancers. Am J Pathol 2015;185:987-1000.

11 Smith $B$, Land $H$. Anticancer activity of the cholesterol exporter ABCA1 gene. Cell Rep 2012;2:580-90.

12 Brown AJ. Cholesterol, statins and cancer. Clin Exp Pharmacol Physiol 2007;34:135-41.

13 Casaburi I, Chimento A, De Luca A, et al. Cholesterol as an Endogenous ERR $\alpha$ Agonist: A New Perspective to Cancer Treatment. Front Endocrinol 2018;9:525.

14 Yamamoto Y, Sasaki N, Kumagai K, et al. Involvement of intracellular cholesterol in temozolomide-induced glioblastoma cell death. Neurol Med Chir 2018;58:296-302.

15 Venkateswaran A, Laffitte BA, Joseph SB, et al. Control of cellular cholesterol efflux by the nuclear oxysterol receptor LXR alpha. Proc Natl Acad Sci U S A 2000;97:12097-102.

16 Yu XH, Qian K, Jiang N, et al. ABCG5/ABCG8 in cholesterol excretion and atherosclerosis. Clin Chim Acta 2014;428:82-8.

17 Zelcer N, Hong C, Boyadjian R, et al. LXR regulates cholesterol uptake through Idol-dependent ubiquitination of the LDL receptor. Science 2009;325:100-4.

18 Chen W, Chen G, Head DL, et al. Enzymatic reduction of oxysterols impairs LXR signaling in cultured cells and the livers of mice. Cell Metab 2007;5:73-9.

19 Bovenga F, Sabbà C, Moschetta A. Uncoupling nuclear receptor LXR and cholesterol metabolism in cancer. Cell Metab 2015;21:517-26.

20 Janowski BA, Willy PJ, Devi TR, et al. An oxysterol signalling pathway mediated by the nuclear receptor LXR alpha. Nature 1996;383:728-31.

21 Vedin LL, Lewandowski SA, Parini P, et al. The oxysterol receptor LXR inhibits proliferation of human breast cancer cells. Carcinogenesis 2009;30:575-9.

22 Vedin LL, Gustafsson JA, Steffensen KR. The oxysterol receptors LXR $\alpha$ and LXRß suppress proliferation in the colon. Mol Carcinog 2013;52:835-44.

23 Clendening JW, Pandyra A, Boutros PC, et al. Dysregulation of the mevalonate pathway promotes transformation. Proc Natl Acad Sci U S A 2010;107:15051-6. 
24 Clendening JW, Penn LZ. Targeting tumor cell metabolism with statins. Oncogene 2012;31:4967-78.

25 Ginestier C, Monville F, Wicinski J, et al. Mevalonate metabolism regulates Basal breast cancer stem cells and is a potential therapeutic target. Stem Cells 2012;30:1327-37.

26 Ostrom QT, Gittleman H, Xu J, et al. CBTRUS statistical report: primary brain and other central nervous system tumors diagnosed in the United States in 2009-2013. Neuro Oncol 2016;18:v1-v75.

27 Villa GR, Hulce JJ, Zanca C, et al. An LXR-cholesterol axis creates a metabolic co-dependency for brain cancers. Cancer Cell 2016;30:683-93.

28 Courtney R, Landreth GE. LXR Regulation of Brain Cholesterol: From Development to Disease. Trends Endocrinol Metab 2016;27:404-14.

29 Hayashi $\mathrm{H}$, Campenot RB, Vance DE, et al. Glial lipoproteins stimulate axon growth of central nervous system neurons in compartmented cultures. J Biol Chem 2004;279:14009-15.

30 Chen J, Zhang X, Kusumo H, et al. Cholesterol efflux is differentially regulated in neurons and astrocytes: implications for brain cholesterol homeostasis. Biochim Biophys Acta 2013;1831:263-75.

31 Facchini G, Ignarro RS, Rodrigues-Silva E, et al. Toxic effects of phytol and retinol on human glioblastoma cells are associated with modulation of cholesterol and fatty acid biosynthetic pathways. J Neurooncol 2018;136:435-43.

32 An Y, Zhang DD, Yu HL, et al. 27-Hydroxycholesterol regulates cholesterol synthesis and transport in C6 glioma cells. Neurotoxicology 2017;59:88-97.

33 Hamm R, Zeino M, Frewert S, et al. Up-regulation of cholesterol associated genes as novel resistance mechanism in glioblastoma cells in response to archazolid B. Toxicol Appl Pharmacol 2014;281:78-86.

34 Yamamoto Y, Tomiyama A, Sasaki N, et al. Intracellular cholesterol level regulates sensitivity of glioblastoma cells against temozolomide-induced cell death by modulation of caspase- 8 activation via death receptor 5-accumulation and activation in the plasma membrane lipid raft. Biochem Biophys Res Commun 2018;495:1292-9.

35 Shojaei S, Alizadeh J, Thliveris J, et al. Inhibition of autophagy by mevalonate pathway inhibitors, a new therapeutic approach to sensitize glioblastoma cells to temozolomide induced apoptosis. Faseb J 2018.

36 Miettinen TP, Björklund M. Mevalonate pathway regulates cell size homeostasis and proteostasis through autophagy. Cell Rep 2015;13:2610-20.

37 Tricarico PM, Romeo A, Gratton R, et al. Lack of prenylated proteins, autophagy impairment and apoptosis in sh-sy5y neuronal cell model of mevalonate kinase deficiency. Cell Physiol Biochem 2017;41:1649-60.

38 Zhang J, Johnson JL, He J, et al. Cystinosin, the small GTPase Rab11, and the Rab7 effector RILP regulate intracellular trafficking of the chaperone-mediated autophagy receptor LAMP2A. J Biol Chem 2017;292:10328-46.

39 Amaya C, Fader CM, Colombo Ml. Autophagy and proteins involved in vesicular trafficking. FEBS Lett 2015;589:3343-53.

40 Longatti A, Lamb CA, Razi M, et al. TBC1D14 regulates autophagosome formation via Rab11- and ULK1-positive recycling endosomes. I Cell Biol 2012;197:659-75.

41 El-Khattouti A, Selimovic D, Haikel Y, et al. Crosstalk between apoptosis and autophagy: molecular mechanisms and therapeutic strategies in cancer. J Cell Death 2013;6:JCD.S11034-55.

42 Ghavami S, Yeganeh B, Stelmack GL, et al. Apoptosis, autophagy and ER stress in mevalonate cascade inhibition-induced cell death of human atrial fibroblasts. Cell Death Dis 2012;3:e330.

43 Gaist D, Hallas J, Friis $\mathrm{S}$, et al. Statin use and survival following glioblastoma multiforme. Cancer Epidemiol 2014;38:722-7.

44 Altwairgi AK, Alghareeb WA, AINajjar FH, et al. Phase II study of atorvastatin in combination with radiotherapy and temozolomide in patients with glioblastoma. J Clin Oncol 2014;32. 